\title{
Thinned Dies in a Stretchable Package
}

\author{
R. Verplancke ${ }^{1,2^{*}}$, T. Sterken ${ }^{1,2}$, D. Cuypers ${ }^{1,2}$, J. Vanfleteren ${ }^{1,2}$ \\ ${ }^{1}$ Centre for Microsystems Technology (CMST) , ELIS Department, Ghent University, Technologiepark $914 \mathrm{a}, 9052$ \\ Zwijnaarde, Belgium \\ ${ }^{2}$ IMEC, Kapeldreef 75, 3001 Leuven, Belgium \\ *E-mail: Rik.Verplancke@UGent.be
}

Tel: +3292645351

\begin{abstract}
Conformable, stretchable electronic circuits relying on conventional high-performance electronic materials have been realized. Thinned dies $(\sim 30 \mu \mathrm{m}$ thickness $)$ are embedded in a slim polyimide package $(50 \mu \mathrm{m}-60 \mu \mathrm{m}$ thickness $)$, electrically connected using polyimide-supported thin-film meandering gold conductors and subsequently embedded in polydimethylsiloxane (PDMS). The technology is demonstrated by embedding a commercially available microcontroller, i.e. a MSP430F1611 (Texas Instruments), thinned down to $\sim 30 \mu \mathrm{m}$ thickness.
\end{abstract}

\section{Introduction}

Electronic circuits which can fully conform and dynamically adapt to arbitrary curved surface topologies (e.g. the human body) are of interest in diverse areas of application. The range of emerging applications is vast and includes biomedical systems such as wearable unobtrusive health monitors (e.g. electrocardiogram (ECG) or temperature sensors) [1] or advanced surgical tools [2] and consumer electronics such as mobile phones which are envisioned to transform their mechanical configuration [3].

To achieve optimal functionality, it is obvious that introducing stretchability in the assembly is highly advantageous. As will be clear from the next section, stretchable electrical interconnections form a key challenge. Two major trends for their realization are reported. One involves the use of primarily organic materials, whereas the other uses conventional high-performance inorganic electronic materials by devising ingenious structural designs to introduce stretchability. A recent review covering these trends can be found in [4].

In this contribution, a strategy based on the latter trend is pursued.

\section{Conformable, Stretchable Electronics Concept}

A general strategy towards conformable, stretchable electronics consists in the use of various non-stretchable platforms hosting electronic components that are connected through stretchable electrical wiring. The platforms are composed of a structural polymer, and are designed to host fragile devices. It is clear that in order to improve the overall flexibility and conformability, a certain degree of flexibility in the platforms themselves is also beneficial.

The stretchable electrical wiring is able to deform with the elastic substrate without compromising the electrical performance. They are realized by patterning thin-film gold metallization in meandering horseshoe shapes (Figure 1), allowing the conductors to function as 'two-dimensional' springs when embedded in an elastomeric substrate such as polydimethylsiloxane (PDMS). The fabrication and mechanical characterization of these interconnections has been described in detail elsewhere [5]. It was found that by introducing polyimide-support around the interconnections, the reliability can be increased greatly. This concept will be used throughout the development of the fabrication process presented in this contribution.

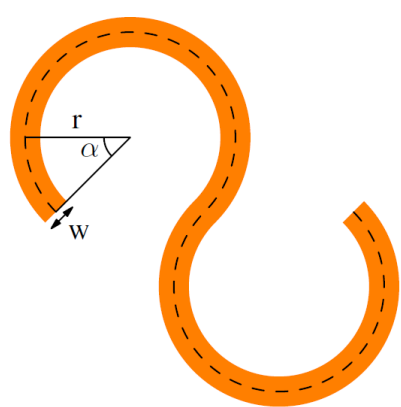

\section{Figure 1: Stretchable electrical interconnections are realized using a sequence of horseshoe shapes.}

A degree of flexibility in the platforms is introduced by embedding electronic components as thinned dies in a slim polyimide package instead of assembling them as bulky offthe-shelf components. The idea underlying this approach is that dies, or any material for that matter, become flexible if sufficiently thinned. This concept has already been reported as an enabler for various applications requiring improved flexibility, e.g. retinal implants or RFID tags [6].

\section{Microcontroller MSP430F1611}

The microcontroller MSP430F1611 from Texas Instruments is used, as it is ideally suited for wearable electronic systems due to its low power consumption. This controller family incorporates a 16-bit RISC Central Processing Unit (CPU), and several peripherals such as digital interfaces $\left(\mathrm{I}^{2} \mathrm{C}\right.$, UART, SPI), timers, analog-to-digital converters (ADCs) and digital-to-analog converters (DACs). Ultra-low power functionality is obtained through the availability of several low power modes, allowing parts of the system to be temporarily disabled.

The microcontrollers are thinned down from $725 \mu \mathrm{m}$ (= standard thickness of an 8 inch silicon wafer) to a target thickness of $30 \mu \mathrm{m}$ by a dicing-before-grinding (DBG) methodology. This was done on wafer scale at Disco; it was found that the dies had thicknesses in the range of $30 \mu \mathrm{m}$ to $40 \mu \mathrm{m}$. The layout is shown in Figure 2. The die measures approximately $4.6 \mathrm{~mm}$ by $4.4 \mathrm{~mm}$. The bond pads $(\sim 75 \mu \mathrm{m}$ by $75 \mu \mathrm{m})$ are distributed over the periphery and are $\mathrm{Ni} / \mathrm{Au}$ surface finished. 


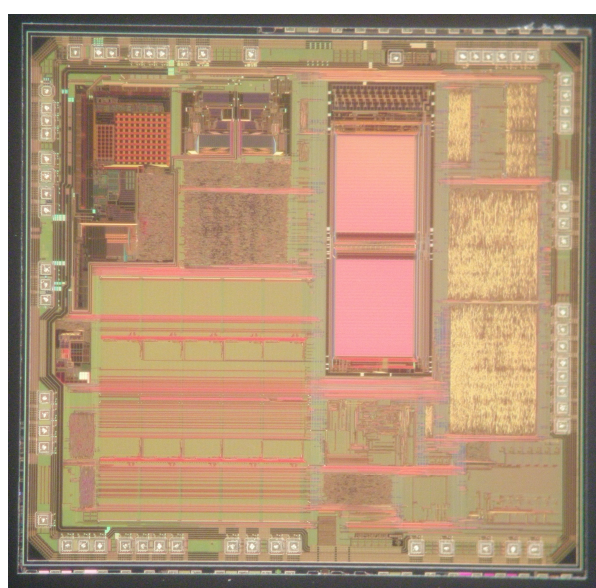

Figure 2: Layout of a MSP430F1611 die.

\section{Fabrication Process}

The fabrication process is schematically represented in Figure 3 , and is briefly discussed.

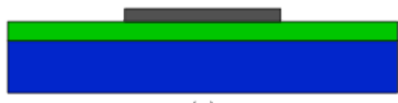

(a)

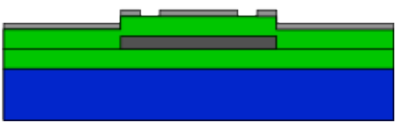

(b)

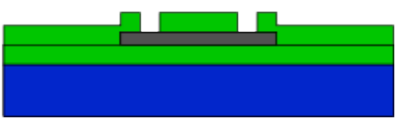

(c)

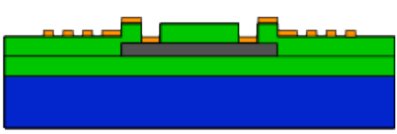

(d)

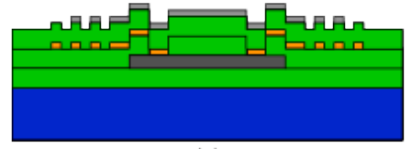

(e)

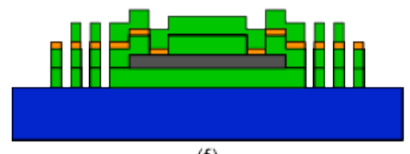

(f)

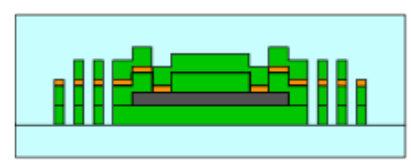

(g)
Float glass $\square$ Polyimide $\square$ Thinned die

Hard Mask $\square$ Gold $\square$ PDMS

Figure 3: Cross-sectional views of the fabrication steps; note that these are not drawn to scale.

Fabrication is performed on 2 inch square glass substrates, and is started by selective application of an adhesion promoter at the edges of the substrate. The adhesion promoter is required to ensure sufficient adhesion of the subsequent polyimide film throughout the fabrication process. Next, a 5.5 $\mu \mathrm{m}$ thick polyimide layer is spin coated and cured at $350{ }^{\circ} \mathrm{C}$ in a nitrogen environment.

As thinned dies often exhibit warpage caused by residual stress of the surface films, care is taken to place the dies flatly on the base polyimide film, in order to avoid voids between the die and the adhesive. This is required in order to avoid breakage of the die and to minimize dimensional errors during subsequent close contact photolithography. Benzocyclobutene
(BCB) is chosen as liquid adhesive. A small volume is dispensed in the center of the substrate and baked for $2 \mathrm{~min}$ at $100{ }^{\circ} \mathrm{C}$ to remove the solvents. The die is then placed with the active side up (face-up) onto the BCB deposit, and the stack is partially cured at $150{ }^{\circ} \mathrm{C}$ while moderate pressure is applied. After removing excess $\mathrm{BCB}$ which has flowed from beneath the die with acetone, the $\mathrm{BCB}$ is fully cured at $250{ }^{\circ} \mathrm{C}$ in a nitrogen atmosphere.

A surface scan of a placed die was taken with an optical profiler WYKO NT3300 (Veeco), and is displayed in Figure 4. As noticed, the die / BCB assembly has a thickness of approximately $40 \mu \mathrm{m}$. In Figure 4(b), the cross-sectional profile of the die along its centerlines is displayed. It is observed that the dies are placed quite flatly, with thickness variations of maximum $5 \mu \mathrm{m}$.

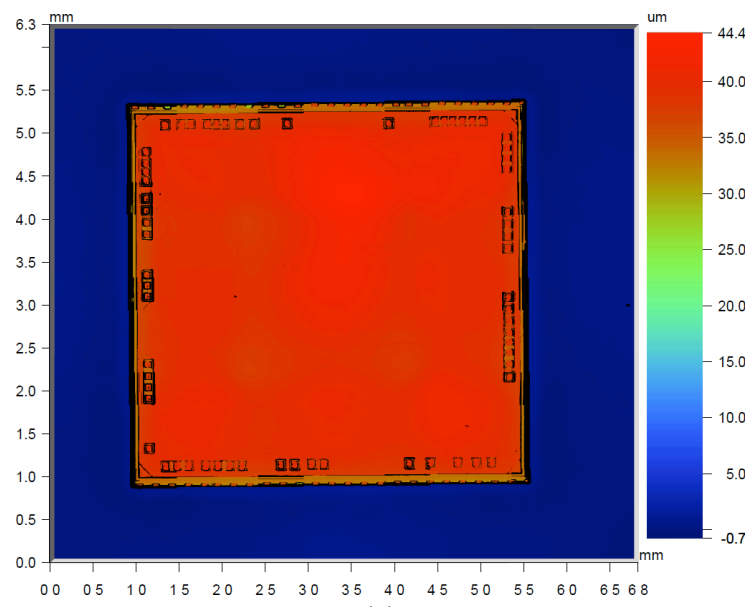

(a)
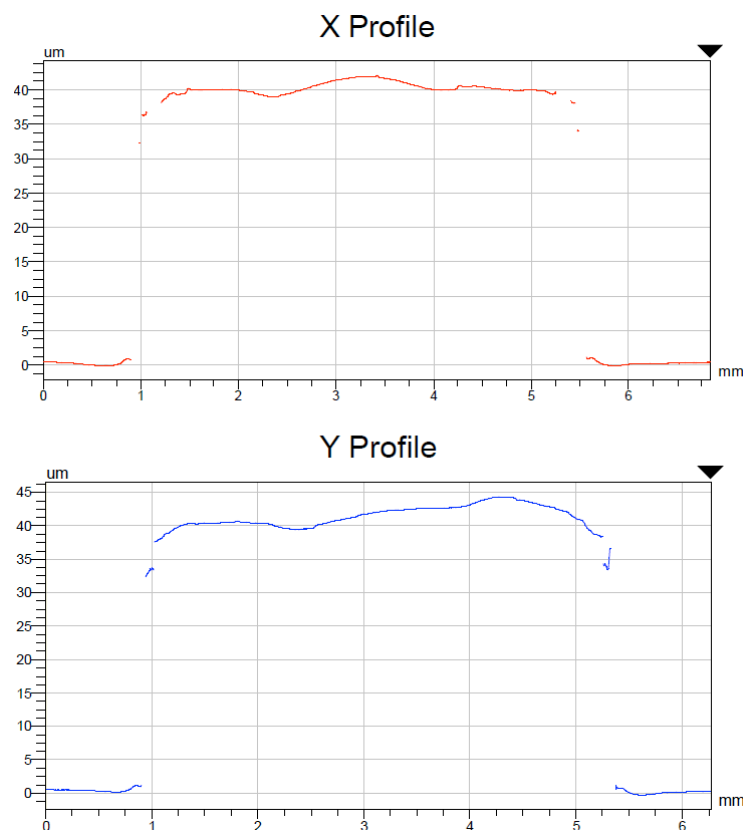

(b)

Figure 4: Surface scan of placed dies: (a) overview; (b) cross-sectional profile of the die along its centerlines.

After placing the die (Figure 3(a)), another polyimide layer is spin coated and cured. The covering polyimide film 
can be as thin as $5.5 \mu \mathrm{m}$ while still resulting in a continuous coating. This is clearly illustrated in the cross-section displayed in Figure 5(a). The polyimide film is then patterned to provide vias towards the die bond pads. To this end, a 300 $\mathrm{nm}$ thick titanium tungsten film is sputter deposited, patterned by photolithography and wet etching (Figure 3(b)), and subsequently used as masking layer to selectively dry etch the covering polyimide film using oxygen $\left(\mathrm{O}_{2}\right)$ and trifluoromethane $\left(\mathrm{CHF}_{3}\right)$ chemistry. The masking layer is then wet etched (Figure 3(c)) and metallization, a $50 \mathrm{~nm}$ thick titanium tungsten adhesion enhancement layer followed by a 200 nm thick gold layer, is sputter deposited. The metallization is patterned through photolithography and wet etching in the desired (stretchable) interconnection design and programming pads (Figure 3(d)). It is clear that covering the die with a $5.5 \mu \mathrm{m}$ thick polyimide layer results in height variations in the order of the thickness of the die. As such non-uniformities are not standard in thin-film processing, special attention is given to the optimization of the photolithography. Two points of concern are monitored: the continuity of the photoresist and hence also the metallization at the edges of the die (Figure 6(a)), and the appearance of shorts around the die due to undeveloped photoresist which remains after insufficient exposure (Figure 6(b)).

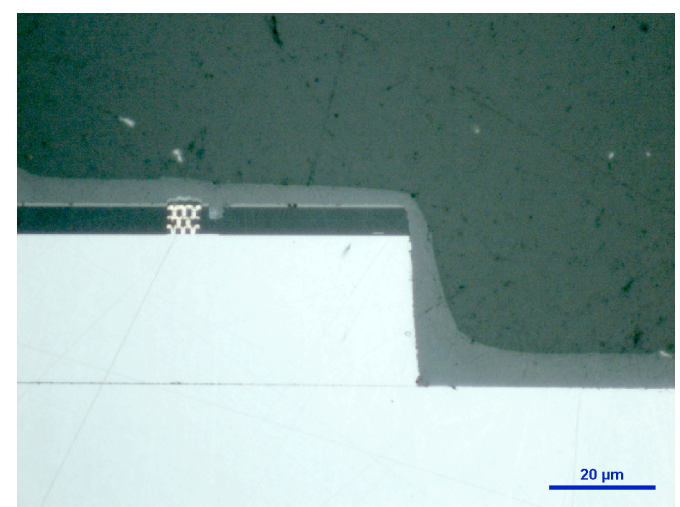

(a)

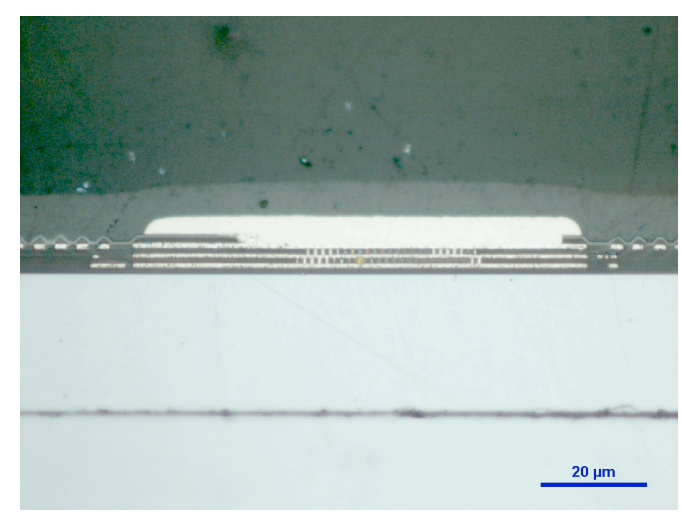

(b)

Figure 5: Cross-section of a $\sim 35 \mu \mathrm{m}$ thick die, placed on a silicon wafer and covered by a $5.5 \mu \mathrm{m}$ thick polyimide film: (a) continuous edge coverage; (b) close-up of a Ni/Au bump.

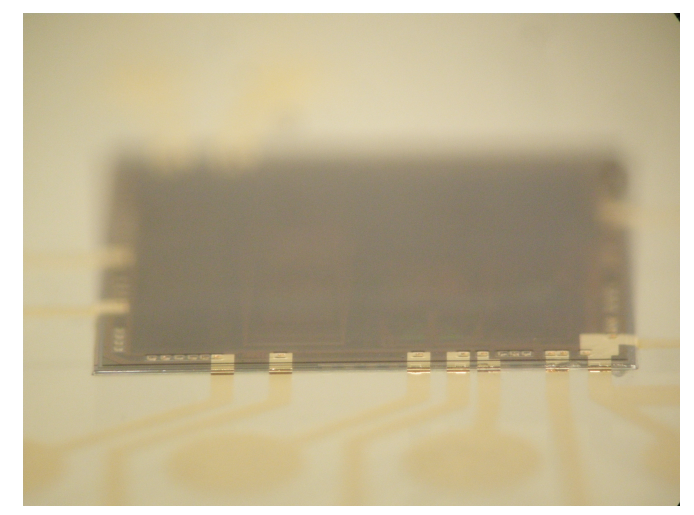

(a)

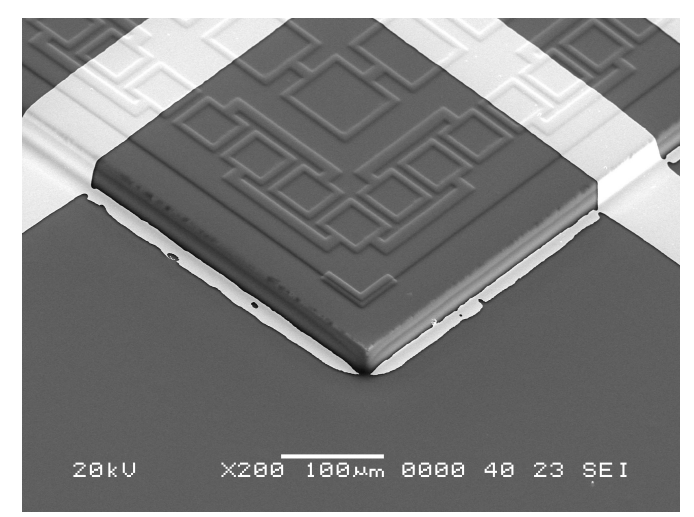

(b)

Figure 6: Optimized photolithography: (a) continuity of the metallization at the edges of the die; (b) shorts around the die due to undeveloped photoresist.

Next, another $5.5 \mu \mathrm{m}$ thick polyimide film is spin coated and cured to cover the metallization, and a $200 \mathrm{~nm}$ thick aluminium hard mask is applied (Figure 3(e)) to allow patterning of the different polyimide layers by dry etching. After stripping the hard mask, polyimide covered stretchable interconnections and polyimide islands hosting the thinned die and footprints for the SMD components are obtained (Figure 3(f)).

The flexible / stretchable circuit is then manually separated from the substrate. This is easily done as the adhesion promoter has only been applied at the edges of the substrate. The overall thickness of the circuit, including the embedded microcontroller, is as low as $60 \mu \mathrm{m}$. At this point, SMD components are assembled, the microcontroller is programmed and the circuit is embedded in PDMS. More information on how this is done for the first demonstrator can be found in the next section.

\section{First Demonstrator}

As already mentioned, the microcontroller MSP430F1611 from Texas Instruments was chosen to build a first simple demonstrator. The design of the demonstrator circuit is displayed in Figure 7. In Figure 7(a), the design for the metallization is displayed, clearly indicating the stretchable interconnections and regions on which SMD components are mounted. In the left bottom corner of the design, also a capacitive touch sensor is observed. In Figure 7(b) the polyimide pattern is displayed, clearly indicating the 
polyimide support for the stretchable interconnections, the square openings to mount SMD components and the circular programming pads.

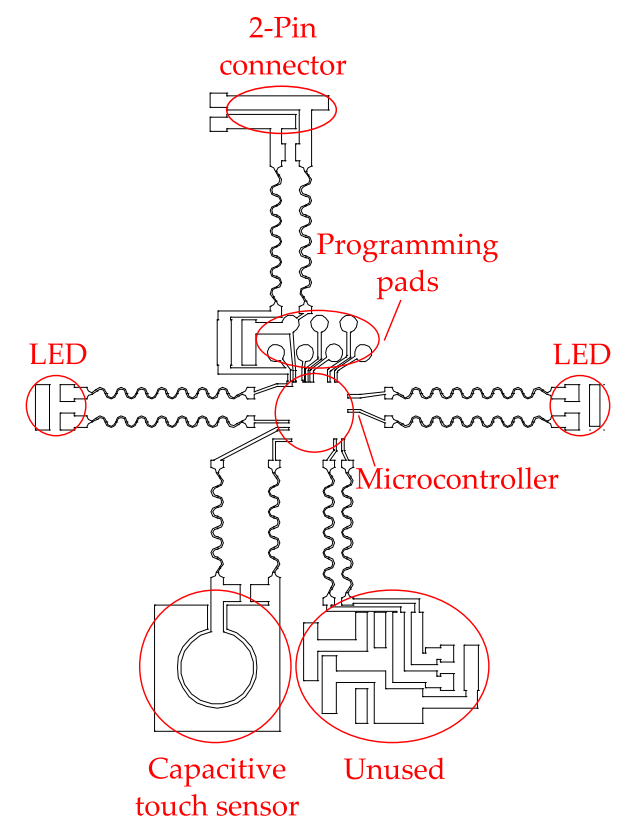

(a)

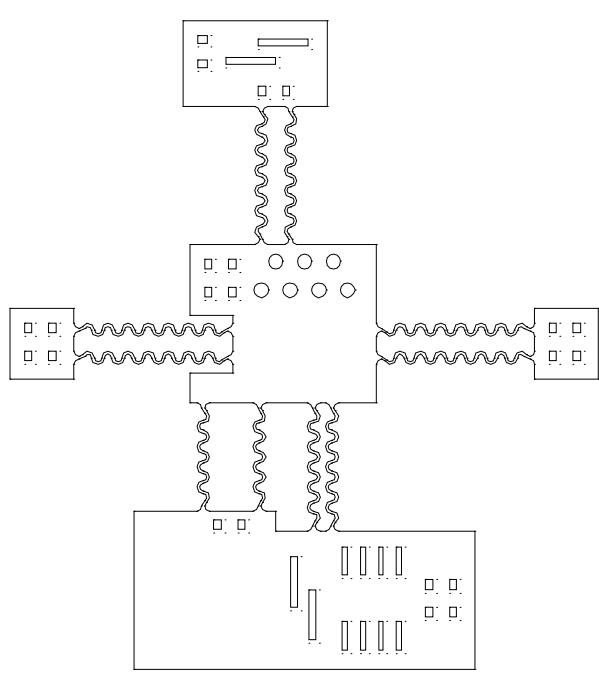

(b)

Figure 7: Design of the first demonstrator: (a) metallization; (b) polyimide pattern.

A released circuit, before embedding in PDMS, is displayed in Figure 8(a). The different SMD components are assembled by means of an isotropic conductive adhesive (ICA) CE-3104 (Emerson \& Cuming). This adhesive is chosen as it is found impossible to perform regular soldering without damaging the gold film because it dissolves too rapidly into the solder.

The microcontroller is programmed using IAR Kickstart software, an OLIMEX MSP430-JTAG-TINY programmer and a homemade PCB providing a pogo pin interface towards the flexible circuit (Figure 9).

In the final stage of the fabrication process, the circuit is embedded in PDMS. Silastic MDX-4 4210 (Dow Corning) was chosen for this purpose. The embedding can be done in several ways: the PDMS can be spin coated after fixating the circuit on a temporary carrier, injection moulding can be performed by inserting the circuit in a dedicated mould, etc. In this case, a $350 \mu \mathrm{m}$ thick PDMS layer was spin coated on a glass substrate, the circuit was carefully placed on top of the liquid PDMS and another $350 \mu \mathrm{m}$ thick PDMS layer was spin coated. The embedded circuit is shown in Figure 8(b). The 2pin connector at the top of the figure is used to provide power to the embedded circuit.

In Figure 10, the functionality of the embedded circuit and the thinned microcontroller is illustrated. The circuit is wrapped around a cylinder with $1.5 \mathrm{~cm}$ curvature radius. Digital I/O and timers are used to blink the LED on the left. The capacitive touch sense library and capacitive touch sensor in the bottom left corner of the design are used to light the LED on the right. Also the DACs, ADCs and integrated temperature sensor were verified to function well.

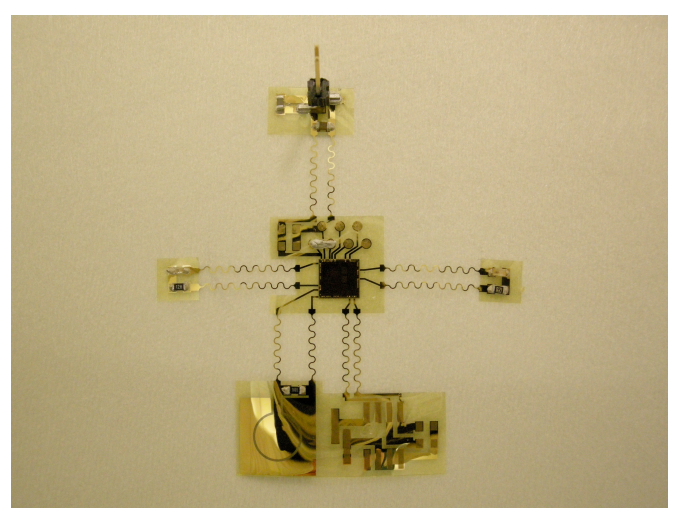

(a)

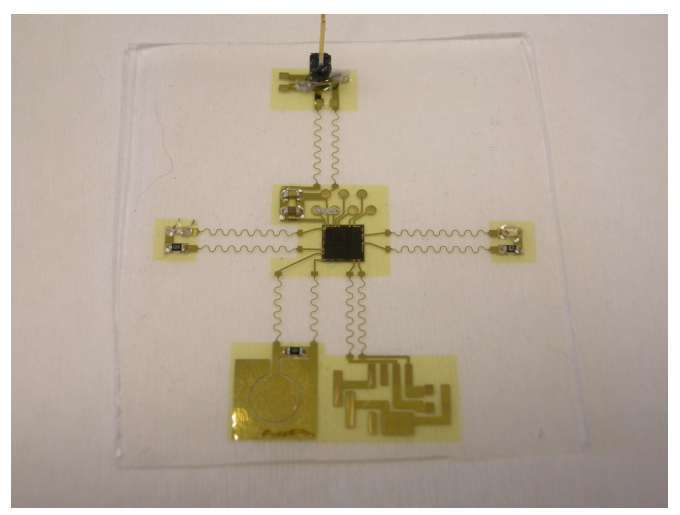

(b)

Figure 8: A released, assembled circuit: (a) before and (b) after embedding in PDMS. 


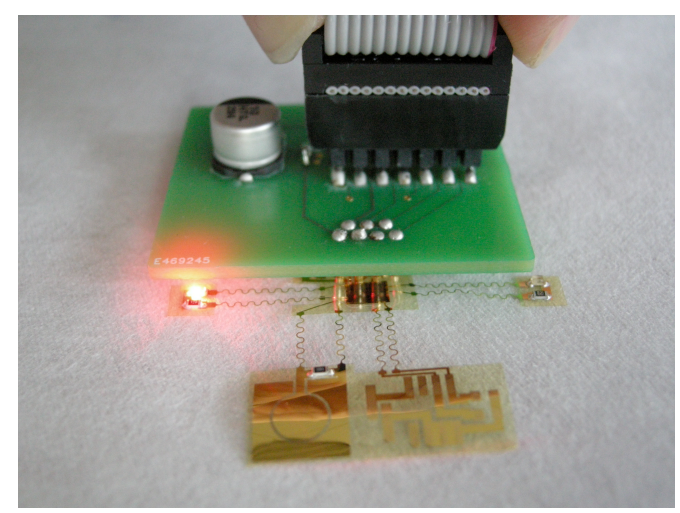

Figure 9: Programming of the microcontroller.

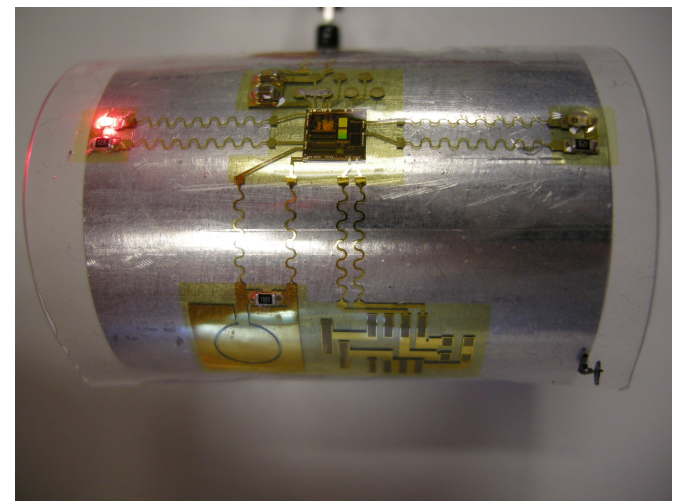

(a)

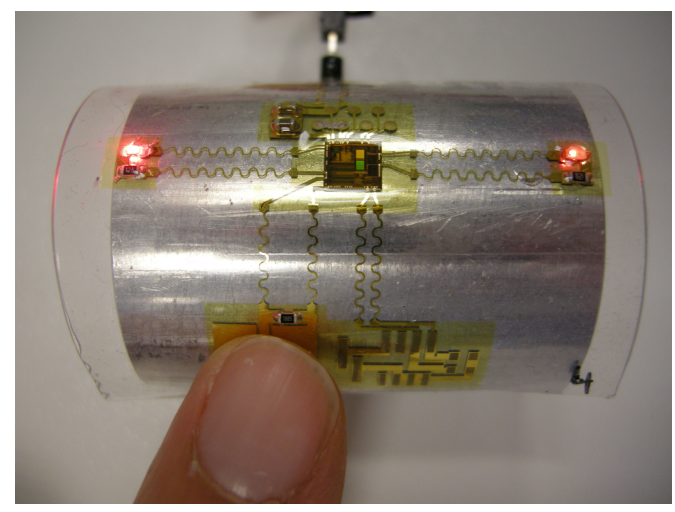

(b)

Figure 10: Embedded circuit wrapped around a cylinder with $1.5 \mathrm{~cm}$ curvature radius.

\section{Conclusions}

In this contribution, we have reported a new conformable, stretchable electronics technology. The technology is based on the use of various non-stretchable platforms hosting electronic components that are connected through stretchable electrical wiring. The stretchable electrical wiring is realized by polyimide-supported thin-film meandering gold conductors, which have been previously shown to be very reliable [5]. Furthermore, flexibility of the platforms is improved by embedding electronic components as thinned dies.

A simple demonstrator is built using a commercially available microcontroller, i.e. a MSP430F1611 (Texas Instruments), thinned down to $30 \mu \mathrm{m}$ thickness. It is shown that the functionality of the microcontroller is maintained when thinned.

\section{Acknowledgments}

The authors would like to thank the Agency for Innovation by Science and Technology in Flanders (IWT) for the financial support through the SBO-BrainSTAR project.

\section{References}

1. Coosemans, J, Hermans, B, Puers, R, "Integrating Wireless ECG Monitoring in Textiles," Sensors and Actuators, Vol. 130-131, (2006), pp. 48-53.

2. Kim, D-H, Lu, N, Ghaffari, R, Kim, Y-S, Lee, S P, Xu, L, Wu, J, Kim, R-H, Song, J, Liu, Z, Viventi, J, de Graff, B, Elolampi, B, Mansour, M, Slepian, M J, Hwang, S, Moss, J D, Won, S-M, Huang, Y, Litt, B, Rogers, J A, "Materials for Multifunctional Balloon Catheters with Capabilities in Cardiac Electrophysiological Mapping and Ablation Therapy," Nature Materials, Vol. 10, No. 4 (2011), pp. 316-323.

3. Ryhänen, T, "Future of Mobile Devices - Energy Efficient Sensing, Computing, and Communication," Symposium on VLSI Circuits, Kyoto, Japan, Jun. 2009, pp. 98-101.

4. Ahn, J-H, Je, J H, "Stretchable Electronics: Materials, Architectures and Integrations," Journal of Physics D: Applied Physics, Vol. 45, No. 10 (2012), 103001.

5. Verplancke, R, Bossuyt, F, Cuypers, D, Vanfleteren, J, "Thin-Film Stretchable Elecontrics Technology Based on Meandering Interconnections: Fabrication and Mechanical Performance," Journal of Micromechanics and Microengineering, Vol. 22, No. 1 (2012), 015002.

6. Burghartz, J N, Appel, W, Harendt, C, Rempp, H, Richter, H, Zimmermann, M, "Ultra-Thin Chip Technology and Applications, a New Paradigm in Silicon Technology," Solid State Electronics, Vol. 54, No. 9 (2010), pp. 818829. 